\title{
The Effects of Acute Stress on Memory, Attention and Cognition in Healthy Human Volunteers
}

\author{
Md. Monir Hossain ${ }^{1}$, Shahnaz Naznin ${ }^{2}$, Dolly Sen $^{2}$ and Mahnaz Hossain Fariba ${ }^{3}$ \\ ${ }^{1}$ Department of Pharmacy, Jagannath University, Dhaka-1100, Bangladesh \\ ${ }^{2}$ Department of Pharmacy, Noakhali Science and Technology University, Sonapur, Noakhali-3814, Bangladesh \\ ${ }^{3}$ Department of Genetic Engineering \& Biotechnology, University of Dhaka, Dhaka-1000, Bangladesh
}

Received: August 08, 2016; Accepted: September 28, 2016; Published (Web): March 19, 2017

\begin{abstract}
The study was designed to investigate the effects of an acute naturalistic stressor, namely, examination of stress on memory, attention and cognition in healthy human volunteers. Fifty-three students participated in this study. All the volunteers were assessed for their neuropsychological states, which are memory, attention and cognition, during non-examination and examination period. The neuropsychological tests used in this study were- logical memory test, digit span test, letter cancellation test, trail making test and Stroop test. It was revealed that there was significant difference $(\mathrm{p}<0.05)$ in the score of logical memory II, backward and total score of digit span, time taken to complete letter cancellation test and score in part C of Stroop test in the exam period. The present study thus demonstrated that acute naturalistic stress improves attention while impairs delayed memory retrieval and cognition.
\end{abstract}

Key words: Acute stress, Neuropsychological test, Memory test, Attention test, Cognition test, Examinations.

\section{Introduction}

The term 'stress', which is originally used to describe a force that exerts physical damage on a structure, is generally defined in biological systems as any condition that seriously perturbs the physiological/psychological homeostasis of an organism. The profound physiological consequences of stress were first shown empirically in Hans Selye's seminal paper of 1936: as "a syndrome produced by diverse nocuous agents” (Selye, 1936, 1973). Stress is a modulator that is potent for learning and memory process. Subsequent researches showed the broad range of adverse physiological effects of stress in animals and humans, including adrenal-gland enlargement, atrophy of thymus and lymph nodes, increased cardiovascular tone, immune system suppression and ulceration (Sapolsky, 1992). Acute stress is a reaction to an immediate threat or perceived threat. Mixed conclusions have been drawn on the effects of acute stress on memory. One view is that acute stress can impair memory while others believe that acute stress can actually enhance memory.
In response to stress, the hypothalamic-pituitary-adrenal axis is activated which leads to an increased secretion of glucocorticoids (GCs) (McEwen, 2000). During stress glucocorticoids and catecholamine such as adrenaline and nor adrenaline are secreted which facilitate adaptation (De Kloet et al., 1998; McEwen, 1998; Sapolsky et al., 2000). These hormones (cortisol in humans; corticosterone in rodents) influence multiple target tissues including the brain. Glucocorticoids have a modulatory influence on memory processes. Several studies have shown that stress and glucocorticoids enhance memory formation while they impair memory retrieval. Studies in rodents have revealed that GCs enhance or impair performance depending on the tested of specific memory tested and on the timing of the stress exposure, respectively (Lupien and McEwen, 1997; De Kloet et al., 1999). Experimental studies in humans have repeatedly shown that GC administration can interfere with performance in working memory as well as declarative memory tasks (de Quervain et al., 2000; Wolf et al., 2001). Studies also revealed that, verbal working memory (Smith et al., 1998) and

Correspondence to: Md. Monir Hossain; Mobile: +8801670117946; E-mail: md.monir.hossain@pharm.jnu.ac.bd 
sources of attention are influenced by corticosteroid levels during the memory process (De Kloet et al., 1998).

In recent decades, an important line of neuroscience researches have shown that stressful experiences can have a negative impact on certain aspect of brain function. Stress has a great impact on memory, attention and cognition. In the present investigation, a study was done to assess the effects of an acute and naturalistic stressor, namely examination, on memory, attention and cognition in healthy human volunteer.

\section{Materials and Methods}

Participants: Undergraduate students who were known to have a period of examinations in the English academic year (i.e., July to December) were approached for this study. Posters advertising the study were displayed in different departments of Noakhali Science and Technology University, Bangladesh. Participation in the project was entirely voluntary. Seventy healthy male and female volunteers, who were adult, were recruited and finally fifty-three volunteers were selected for taking part in the experiment. Written informed consent was obtained from each participant prior to study. Before the selection of the volunteers, they were introduced with a complete set of medical health questions to evaluate their health conditions for the suitability of the study. All subjects were interviewed and included in the study only when they met the following criteria: 1) no history of neuropathological conditions or illness, 2) absence of hospitalization for psychiatric illness, 3) no history of drug or alcohol abuse, 4) normal psychomotor development. Those who had diabetes, hypothyroidism, renal disease or malignancy or were on thiazide diuretics, $\beta$-blockers or corticosteroids; had admission to hospital with a severe illness within the previous 3 months were also excluded. We also excluded those with a known cardiovascular condition (ischemic heart disease, peripheral arterial disease, abdominal aortic aneurysm, and carotid artery disease). All the habitual smokers who usually smoke more than eight cigarettes per day were dropped from the selection procedure of volunteers. Subjects were advised to follow standard diet during the study period and were asked to avoid caffeine twelve hours prior the study. The educational background and body-mass were also considered during the selection of participants so that the differences among the selected participants can be minimized. Finally, fifty-three participants who were divided into two groups (non-exam and exam), participated in the memory, attention and cognition tests reported here.

Treatment procedure: The study was conducted over an exam period. The volunteers were divided into two groups. One group (group A) was non-examinee. The second group (group B) was taking examinations for the same period of time in the similar fashion. The assessment was done to measure the condition of memory, attention and cognition. For all the volunteers, the sequences of the tests administered were kept constant. To estimate health conditions during the study period, age, weight, height, education year, IQ test and blood pressure were measured. The demographic information of the volunteers did not vary much statistically (Table 1).

\section{Test of memory:}

Logical memory (LM): Logical memory test, a subtest of the Wechsler memory scale, was used to measure immediate memory and delayed memory (Wechsler, 1997). In the Logical memory, the volunteers were told a brief story and then they were asked to retell the story twice: once immediately upon hearing the story (Logical Memory I, LM-I), and a second time after a 30 min delay (Logical Memory II, KM-II). During the test, story elements the participants used in each of their retellings were noted. The score was then calculated by counting the number of elements used in a volunteer's retelling.

Digit span test (DST): The Digit Span test (DST) (subtest of the Wechsler intelligence scale) (Wechsler, 1987) was used to assess simple verbal working memory. The test consists of two parts, digits forward (DSTF) and digit backward (DSTB). Strings of digits were read aloud (e.g., 248 ) to the participants at a rate of one digit per second (each string increasing in length from three to nine digits in forward test and two to eight digits in backward test). After every string, the participants were asked to repeat the string. Each subtest (forward and backward) was stopped when a 
participant incorrectly reproduced two successive strings or when a full digit number has been successfully repeated. The total score of the digit span test (DSTT) is the sum of the maximal digit numbers that a participant can recall from forward and backward testing.

\section{Attention test:}

Letter Cancellation Test (LCT): Letter cancellation test (LCT) was used for assessing visual search, scanning and attention (Benton, 1968). It consists of rows of letters randomly interspersed with a designated target letter. The participants were asked to cross out all the target letters. Performance was scored for a subject according to the number of correct responses (LCTC) and the time taken to complete the test (LCTT).

Trail Making Test (TMT): Trail making test (TMT) (Reitan and Wolfson, 1985) provides information on visual search, scanning, speed of processing, attention, mental flexibility and executive functions. TMT, in this investigation, consists of two parts- TMTA and TMTB. TMTA requires drawing lines sequentially connecting 25 encircled numbers distributed on a sheet of paper. Task requirements are similar for TMTB except the fact that the person must alternate between numbers and letters (e.g., 1, A, 2, B, 3, C, etc.). The score on each part represents the amount of time required to complete the task.

Cognition test: Stroop test (ST): The Stroop colorWord test-Victoria version (VST) (Regard, 1981) was used to measure of selective attention and cognitive flexibility which was originally developed by Stroop (Stroop, 1935). The test had three parts. In part D (Dots) [STD], the participants were asked to tell the names of the colors of 24 dots printed in blue, green, red and yellow as quickly as possible. Part W (Words) [STW] is similar to Part D, except that the Dots are replaced by common words printed in lowercase letters. The participants were asked to tell the name of the colors in which the stimuli are printed and to disregard their verbal content. Part C (Colors) [SDC] is similar to parts $\mathrm{D}$ and $\mathrm{W}$, but here the colored stimuli were the color names 'blue, green, red and yellow' printed lowercase so that the printed color never corresponds to the color name. For each part, the time to complete and the number of errors were recorded. Spontaneous corrections were scored as correct.

Statistical analysis: Data were expressed as mean \pm standard error. Statistical analysis was performed using the statistical software package SPSS. Independent sample t-test was used to determine the level of significance between non-examinee and examinee group.

\section{Results}

Test of memory: Logical Memory: There is no significant $(\mathrm{p}<0.05)$ difference observed in Logical memory-I between the two groups but in logical memory-II there was significant decrease $(p<0.05)$ in score for the group attending examinations (Table 2).

Digit Span: In the DST, there was not any statistical difference in digit forward $(\mathrm{p}>0.05)$ score in group A and group B. However, there was decrease $(\mathrm{p}>0.05)$ in score in backward test hence, in the total score of the exam group due to stress (Table 2).

Table 1. Demographic information of the volunteers.

\begin{tabular}{ccccccccc}
\hline Group & $\begin{array}{c}{ }^{\mathrm{a}} \text { Age } \\
\text { (Year) }\end{array}$ & $\begin{array}{c}{ }^{\mathrm{a}} \text { Weight } \\
(\mathrm{Kg})\end{array}$ & $\begin{array}{c}{ }^{\mathrm{a}} \text { Height } \\
(\text { Meter })\end{array}$ & $\begin{array}{c}{ }^{\mathrm{a}} \text { Systolic } \\
\mathrm{BP}(\mathrm{mm} \\
\mathrm{Hg})\end{array}$ & $\begin{array}{c}{ }^{\mathrm{a}} \text { Diastolic } \\
\text { BP (mm Hg) }\end{array}$ & $\begin{array}{c}{ }^{\mathrm{a}} \text { Education } \\
(\text { Year } \\
\text { Completed })\end{array}$ & $\begin{array}{c}{ }^{\mathrm{a}, \mathrm{b}} \mathrm{BMI} \\
\left(\mathrm{Kg} / \mathrm{m}^{2}\right)\end{array}$ & $\begin{array}{c}{ }^{\mathrm{a}, \mathrm{c}} \text { Estimated } \\
\mathrm{IQ}\end{array}$ \\
\hline Group-A & $20.7 \pm 0.2$ & $66.7 \pm 0.8$ & $1.6 \pm 0.0$ & $114.1 \pm 1.0$ & $82.6 \pm 0.8$ & $13.8 \pm 0.2$ & $25.0 \pm 0.3$ & $117.9 \pm 0.8$ \\
Group-B & $20.8 \pm 0.2$ & $67.5 \pm 1.0$ & $1.7 \pm 0.0$ & $114.8 \pm 0.9$ & $81.5 \pm 1.1$ & $13.8 \pm 0.2$ & $24.7 \pm 0.2$ & $118.6 \pm 0.6$ \\
\hline
\end{tabular}

${ }^{\mathrm{a}}$ Mean \pm Standard Error; ${ }^{\mathrm{b}} \mathrm{BMI}=$ Body Mass Index; ${ }^{\mathrm{C}} \mathrm{IQ}$ was estimated from National Adult Reading Scale. 
Table 2. Neuropsychological test.

\begin{tabular}{|c|c|c|c|c|}
\hline \multicolumn{3}{|c|}{ Neuropsychological Tests } & \multirow{2}{*}{$\begin{array}{c}\text { Group A ( } \mathrm{n}=28 \text {, value expressed } \\
\text { as mean } \pm \text { SE) } \\
15.2 \pm 0.3\end{array}$} & \multirow{2}{*}{$\begin{array}{c}\text { Group B ( } \mathrm{n}=25 \text {, value expressed } \\
\text { as mean } \pm \mathrm{SE}) \\
14.8 \pm 0.2\end{array}$} \\
\hline \multirow{5}{*}{ 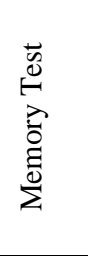 } & \multirow[t]{2}{*}{ Logical Memory } & Logical Memory-I & & \\
\hline & & Logical Memory-II & $13.3 \pm 0.2$ & $11.7 \pm 0.2^{*}$ \\
\hline & \multirow[t]{3}{*}{ Digit Span } & Forward & $5.4 \pm 0.1$ & $5.4 \pm 0.1$ \\
\hline & & Backward & $4.2 \pm 0.1$ & $3.7 \pm 0.1^{*}$ \\
\hline & & Total & $9.6 \pm 0.1$ & $9.1 \pm 0.1^{*}$ \\
\hline \multirow{4}{*}{ 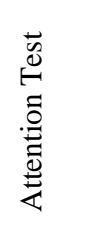 } & \multirow{2}{*}{$\begin{array}{l}\text { Letter } \\
\text { Cancellation } \\
\text { Test }\end{array}$} & Correct Response & $116.9 \pm 0.3$ & $117.0 \pm 0.4$ \\
\hline & & Time (second) & $115.1 \pm 1.1$ & $105.2 \pm 1.5^{*}$ \\
\hline & \multirow{2}{*}{$\begin{array}{l}\text { Trail Making } \\
\text { Test (TMT) }\end{array}$} & TMT-A & $80.5 \pm 1.3$ & $78.8 \pm 1.0$ \\
\hline & & TMT-B & $219.5 \pm 4.4$ & $211.0 \pm 3.2$ \\
\hline \multirow{3}{*}{ 官 } & \multirow[t]{3}{*}{ Stroop Test } & Part D-Time & $17.6 \pm 0.2$ & $17.6 \pm 0.3$ \\
\hline & & Part W-Time & $16.7 \pm 0.2$ & $16.4 \pm 0.2$ \\
\hline & & Part C-Time & $22.2 \pm 0.4$ & $24.0 \pm 0.2^{*}$ \\
\hline
\end{tabular}

$\mathrm{SE}=$ Standard Error of Mean. ${ }^{*}$ statistically significant in comparison to baseline data where $\mathrm{p}<0.05$. The unit of time was second.

Attention test: Letter cancellation test: In LCT, statistical difference ( $\mathrm{p}>0.05$ ) was not observed in the score for number of correct responses for both of the groups. However, significant decrease $(p<0.05)$ was observed statistically in the time taken to complete the test in the group which consisted of examinees (Table 2).

Trail making test: In both TMT-A and TMT-B, there was no any statistically significant difference $(\mathrm{p}<0.05)$ in the time required to make the trail for both of the groups (Table 2).

\section{Cognitive test:}

Stroop test: There was not any significant statistical variation $(\mathrm{p}>0.05)$ in part $\mathrm{D}$ and part $\mathrm{W}$ of Stroop test conducted in both of the groups. However, difference $(\mathrm{p}<0.05)$ was found in the score of part $\mathrm{C}$ in both of the groups (Table 2).

\section{Discussion}

The present investigation was conducted to assess the effect of an acute and naturalistic stressor, i.e., examinations on memory, attention and cognition in young human subjects. The obtained data revealed that the examination period was associated with a significant increase in self-reported levels of stress. A significant negative effect of acute stress on delayed memory retrieval and cognition was observed while there is a significant increase in attention. The LM-I number of correct response in Letter Cancellation Test, TMT-A, TMT-B, the score of Part D and Part W in Stroop test were not significant. Other tasks namelyLM-II in logical memory test, time taken to cancel all the letters in LCT, score of part C in Stroop test were observed to have relatively changed and the values were significantly different due to Stress. Systolic and diastolic pressure of the volunteers was documented during the study period and difference could not be found between both the groups.

Cortisol is widely regarded as an objective marker of changes in psychological stress (Kirschbaum et al., 1996) and has been shown to increase during periods of both acute (Al'Absi et al., 1997) and chronic (Vedhara et al., 1999) stress. Other investigation suggests that an increase in self-reported levels of stress reduced the cortisol level and was associated with impaired cognitive function (Vedhara et al., 2000). Moderate cortisol elevation in response to psychosocial stress, most likely in combination with activation of the autonomous nervous system, can lead to negative effects on delayed memory (Kuhlmann et at., 2005). 
Examination in student life can cause stress on an individual. This study validates the examination as a naturalistic stressor on students in Bangladeshi population.

However, it would appear reasonable to speculate that confounding factors may include: seasonal variations in cortisol levels; the activity of other stress hormones (e.g., adrenalin, noradrenalin); the unique nature of examination stress (i.e., a predictable and often recurring stressor); characteristics of the target population (i.e., students) or participants' appraisal processes. There have been data suggesting that cortisol levels are higher during the months in winter compared to the months in summer (Maes et al., 1997). It is not clear whether the interval between our non-exam and exam sampling periods may have been influenced by such seasonal variations, but this factor may have contributed to decline delayed memory and cognition during the exam period. A limitation of the current study is the missing information of women's menstrual cycle. Furthermore, age might have an influence on the association between cortisol and memory.

To conclude, in this experiment we observed a significant negative effect of acute stress on delayed memory and cognition. As previous investigation showed that examination stress does not reliably produce increases in cortisol. Indeed, the increase in self-reported levels of stress during the examination period might be associated with an unexpected decrease in levels of cortisol. As a consequence, the origins of the observed cognitive changes between the non-exam and exam periods remain unclear. Further detailed studies are needed to evaluate the actual effect of glucocorticoid on memory, attention and cognition on stress condition.

\section{Conclusion}

The experimental reports evidenced that significant increase in self-reported levels of stress is caused by examinations. It can be concluded that acute stress caused by examinations have a significant negative impact on delayed memory retrieval and cognition while there is a significant increase in attention.

\section{Acknowledgements}

The authors express deep gratitude to the students of Noakhali Science and Technology University for participating in the research work as volunteers. Their valuable contributions led to the completion of the work successfully.

\section{Conflict of Interest}

The authors declare that they have no personal or financial conflict of interest.

\section{References}

Al'Absi, M., Bongard, S., Buchanan, T., Pincomb, G.A., Licinio, J. and Lovallo, W.R. 1997. Cardiovascular and neuroendocrine adjustment to public speaking and mental arithmetic stressors. J. Psychophysiol. 34, 266275.

Benton, A.L. 1968. Differential behavioral effects in frontal lobe disease. Neuropsychologia. 6, 53-60.

De Kloet, E.R., Vreugdenhil, E., Oitzl, M.S. and Joels, M. 1998. Brain corticosteroid receptor balance in health and disease. Endocr. Rev. 19, 269-301.

De Kloet, E.R., Oitzl, M.S. and Joels, M. 1999. Stress and cognition: are corticosteroids good or bad guys? Trends. Neurosci. 22, 422-426.

de Quervain, D.J., Roozendaal, B., Nitsch, R.M., McGaugh, J.L. and Hock, C. 2000. Acute cortisone administration impairs retrieval of long-term declarative memory in humans. Nat. Neurosci. 3, 313-314.

Kirschbaum, C., Wolf, O.T., May, M., Wippich, W. and Hellhammer, D.H. 1996. Stress- and treatment-induced elevations of cortisol levels associated with impaired declarative memory in healthy adults. Life. Sci. 58, 14751483.

Kuhlmann, S., Piel, M., Wolf, O.T. 2005. Impaired memory retrieval after phychosocial stress in healthy young men. J. Neurosci. 25, 2977-2982.

Lupien, S.J. and McEwen, B.S. 1997. Then acute effects of corticosteroids on cognition: integration of animal and human model studies. Brain. Res. Rev. 24, 1-27.

Maes, M., Mommen, K., Hendrickx, D., Peeters, D., D’Hondt, P., Ranjan, R., De Meyer, F. and Scharpe, S. 1997. Components of biological variation, including seasonality, in blood concentrations of TSH, TT3, FT4, PRL, cortisol and testosterone in healthy volunteers. Clin. Endocrinol. 46, 587-598.

McEwen, B.S. 1998. Protective and damaging effects of stress mediators. N. Engl. J. Med. 338, 171-179. 
McEwen, B.S. 2000. The neurobiology of stress: From serendipity to clinical relevance. Brain. Res. 886, 172189.

Regard, M. 1981. Cognitive rigidity and flexibility: A neuropsychological study. Unpublished Ph.D. dissertation, University of Victoria.

Reitan, R.M. and Wolfson, D. 1985. The Halstead-Reitan Neuropsychological Test Bettery. Tucson, Ariz., Neuropsychology Press.

Sapolsky, R.M. 1992. Stress, the Aging Brain, and the Mechanisms of Neuron Death. Cambridge, Massachusetts, MIT Press.

Sapolsky, R.M., Romero, L.M. and Munck, A.U. 2000. How do glucocorticoids in- fluence stress responses? Integrating permissive, suppressive, stimulatory, and preparative actions. Endocr. Rev. 21, 55-89.

Selye, H. 1936. A syndrome produced by diverse nocuous agents. Nature 138, 32.

Selye, H. 1973. The evolution of the stress concept. Am. Psychol. 61, 692-699.

Smith, E.E., Jonides, J., Marshuetz, C. and Koeppe, R.A. 1998. Components of verbal working memory: Evidence from neuroimaging. Proc. Natl. Acad. Sci. 95, 876-882.
Stroop, J.R. 1935. Studies of interference in serial verbal reaction. J. Exp. Psychol. 18, 643-662.

Vedhara, K., Cox, N.K.M., Wilcock, G.K., Perks, P., Hunt, M., Anderson, S., Lightman, S.L. and Shanks N.M. 1999. Chronic stress in elderly carers of dementia patients and antibody response to influenza vaccination. Lancet. 353, 627-631.

Vedhara, K., Hyde, J., Gilchrist, I.D., Tytherleigh, M., Plummer, S. 2000. Acute stress, memory, attention and cortisol. Psychoneuroendocrinology. 25, 535-549.

Wechsler, D. 1987. Manual for the Wechsler Adult Intelligence Scale-Revised, The Psychological Corporation., New York, pp. 29-31.

Wechsler, D. 1997. Administration and scoring manual for the Wechsler Adult Intelligence Scale. 3rd ed. San Antonio, TX, Psychological Corporation.

Wolf, O.T., Schommer, N.C., Hellhammer, D.H., McEwen, B.S. and Kirschbaum, C. 2001. The relationship between stress induced cortisol levels and memory differs between men and women. Psychoneuroendocrinology 26, 711-720. 